\title{
CONTINUATION OF DISCUSSION
}

BOLSHAAR DISCUSSION, Continued from page 22

the stars in my sample appear to exhibit a negative luminosity effect in the $\mathrm{Na}$ ' $\mathrm{D}$ ' lines. Mould's model atmosphere analysis suggests that this effect is due to pressure broadening. I tend to agree.

LIEBERT: George Wallerstein has likened the search for a true $\mathrm{K}, \mathrm{M}$ abundance subdwarf to the search for the 'Holy Grail'. But we must remember here that, even though the high velocity M dwarfs are easily selected out in surveys based on proper motion or reduced proper motion, these stars must be found within a much more restricted space volume about the Sun than the F and G stars. You have only a handul of high velocity stars, I believe, in your sample and there aren't a great many more $M$ stars known with either high velocity or these spectral peculiarities. Yet only a small fraction of halo stars would be expected to have [Fe/H] $\sim-2$.

I wonder if we should expect to have any really extreme cases in our current sample.

$B O E S H A A R$ : Very few. I agree.

BLANCO, HOAG AND McCARTHY DISCUSSION, Continued from page 38

a group of low dispersion spectroscopists that 'the best auxiliary equipment for a low dispersion spectral study is a fine slit spectrograph with high dispersion'. We look to slit spectroscopy for further illumination of our results.

\section{FLOWER DISCUSSION, Continued from page 154}

0.10. So, you can change both $\mathrm{Z}$ and $\mathrm{Y}$, and change the modulus.

KÄHLER: You mentioned the uncertainty of the opacities. In addition one should consider the uncertainty of the burning rates. There might be undetected resonances in the CNO-cycle which could be important.

WEIDEMANN: Are you aware of our paper (Koester and Weidemann, Astron. Astrophys. 25, 437, 1973) in which we discussed the chemical composition and the location of the Hyades main sequence in the color magnitude and mass-luminosity diagrams? We used older models, by Copeland et al. (1970). How would your results change, had you used those models?

FLOWER: Yes, I am but their models only go up to about $2.5 \mathrm{M}_{\Theta}$ so I cannot compare them with my upper main sequence.

$C O X$ : Can you tell us whether the ratio of mixing length to scale height affects the results you have given?

FLOWER: Since my main sequence models are hotter than about $\log \mathrm{T}_{\mathrm{eff}}=4.0$, the models are radiative. This is, of course, an advantage, because comparisons with the upper main sequence minimizes the uncertainties in the mixing length theory, as well as, uncertainties in line blanketing and, possibly, in abundance effects on temperature scales.

\section{ABT AND LEVATO DISCUSSION, Contined from page 156}

is unusually good for detecting faint peculiarities. Hartoog did not have a similar advantage. Otherwise the results would probably have been the same.

MORGAN: What is the metallic line type of those broad-lined Am stars in the Orion association?

$A B T$ : They're early A-stars like Sirius.

\section{HIRSHFELD, MCCLURE AND TWAROG DISCUSSION, Continued from page 166}

factor, not age. This, of course, still permits the original picture that the heavy element abundance greatly increased with time during the earlier phases of the Galaxy, but in such a way as to produce the present position-dependent abundances.

$D E M I N G$ : I notice that you adopt an approximately solar metallicity for M67. However the CN strength of M67 is fairly high, about halfway between the Hyades and the average value for the solar neighborhood. Are you prepared to claim that M67 may be somewhat CNO-rich?

MCCLURE: No. I'm sure that the difference in inferred abundance on the basis of UV excess and on CN strength is less than the errors of observation.

\section{5}

A. G. Davis Philip and D. S. Hayes (eds.), The HR Diagram, 405-406. All Rights Reserved.

Copyright $\odot 1978$ by the $I A U$ 
NORRIS DISCUSSION, Continued from page 204

You see the width in (B-V) because it reflects the blanketing due to the Fe peak metals that cover the spectrum. But if you had the corresponding variations in the CNO group that were primordial, the question arises whether you would get much spread in the giant branch.

NORRIS: In our (Bessell and I) investigation in the $\mathrm{CN}$-strong stars in $\omega$ Cen we found a deep depression at $4200 \AA$. There also seems to be a depression stretching blueward of $4000 \AA$, due to some unknown molecule.

We thought that we could explain a large part by some blocking in the B-band, while the V-band does not contain $\mathrm{CN}$ or any thing like that.

DICKENS: The feature at $4215 \AA$ seems enhanced in one of your spectra of a $\mathrm{CN}$-strong star in 47 Tuc. Whilst this could perhaps be caused by the $\mathrm{CN}$ itself in this star, it would be interesting to investigate the possibility of $\mathrm{Sr}$ enhancements in the stars which show $\mathrm{CN}$ enhancement, particularly on the AGB, by looking at other lines of the Sr multiplet. Some stars in $\omega$ Cen showed simultaneous enhancements of N (H-burning) and $\mathrm{Sr}$ and $\mathrm{Ba}$ (S-process) which are difficult to understand theoretically.

NORRIS: Our computations suggest that the feature at $4215 \AA$ increases because of $\mathrm{CN}$ enhancement. The high dispersion spectra (obtained with the Anglo Australian Telescope) which we have in this region run from $4120-4600 \AA$, so that we do not have the possibility of commenting on the Sr II line at $4077 \AA$.

\section{PHILIP DISCUSSION, Continued from page 220}

constant $\mathrm{g} \theta^{4}$ yielding constant $\mathrm{M} / \mathrm{L}$. You have models subject to only small differences depending on helium content. Consistent with the cosmologically produced helium wouldn't you derive a reliable mass? Your stars would help determine the helium core mass of a red giant of the old halo population.

PHILIP: Yes. In a paper in preparation concerning the field and globular cluster HB stars, masses will be calculated. However, I have not yet made these calculations.

\section{RENZINI DISCUSSION, Continued from page 228}

KRAFT: There are other ways to get the spread on the HB, but if (and that's a big 'if') there are primordial abundance variations in globular clusters, then they will directly cause a spread on the HB without appeal to rotation, although rotation certainly should be considered.

\section{LUB AND PEL DISCUSSION, Continued from page 236}

COX: Let me be sure that you have not solved the mass anomaly for the bump or double mode Cepheids.

PEL: Yes, what I said refers only to the general mass-problem with all Cepheids. Our data do not change the mass-discrepancies of bump-Cepheids and double-mode Cepheids.

MADORE: There does exist one other independent and totally fundamental method for determining the masses of Cepheids: the use of binary systems. Purely photometric means exist for discovering Cepheid binaries (Madore, B. F., Mon. Not. R. Astron. Soc., 1976) and one of the brightest candidates, SU Cygni, has been found to be a spectroscopic binary. A preliminary orbit has been derived and a lower limit to the Cepheid mass has been obtained. This lower limit is already consistent with the evolutionary masses and is higher than the anomalously low pulsation mass. 